\title{
Medium-Term Results of Balloon Valvuloplasty of Native Pulmonary Valve Stenosis with and without Supravalvular Obstruction in Childhood
}

\author{
Olivia Lenoir $^{1,2}$, Daniel Quandt ${ }^{1,2}$, Oliver Kretschmar ${ }^{1,2}$ and Walter Knirsch ${ }^{1,2, *}$ \\ ${ }^{1}$ Pediatric Cardiology, Pediatric Heart Center, Children’s Research Center, University Children’s Hospital Zurich, Zurich, \\ Switzerland \\ ${ }^{2}$ University of Zurich, Zurich, Switzerland \\ *Corresponding Author: Walter Knirsch. Email: walter.knirsch@kispi.uzh.ch
}

Received: 30 March 2021 Accepted: 19 August 2021

\begin{abstract}
Objectives: Factors influencing results of balloon valvuloplasty (BVP) of pulmonary valve stenosis (PS) in children are investigated. Background: BVP has become the standard of care for PS, medium-term results are not uniform and depend on various preconditions. Methods: We analysed the medium-term results of BVP of PS in children in an observational, single centre study. Need for additional procedure was defined as outcome after initial BVP. Results: We included 143 children ( 83 female) at a median (IQR) age of $2.6(0.26-9.24)$ months and body weight of 5 (3.4-8) $\mathrm{kg}$ at BVP with a follow-up of 5.04 (1.6-10.2) years. We used balloon size of $10(9-14) \mathrm{mm}$ and maximal balloon pressure of $4(3.5-10)$ atm, resulting in balloon-to-pulmonary annulus ratio of 1.28 (1.2-1.4). Systolic pressure gradient of PS was reduced with BVP $(43.5 \mathrm{mmHg} v s .14 .0 \mathrm{mmHg}, p<0.001)$ and confirmed by echocardiography $(68.0 \mathrm{mmHg} v s .25 .0 \mathrm{mmHg}, p<0.001)$ day 1 post procedure. Pulmonary BVP with associated supravalvular PS resulted in a relevant reduction of systolic pressure gradient in 23 of 31 patients $(74.2 \%)$. Early additional procedure was necessary in 14 patients (9.8\%) after $0.2(0.1-0.7)$ years due to residual PS $(n=13)$ and infective endocarditis $(n=1)$. Factors for additional procedures were associated supravalvular PS with a higher residual pressure gradient, but not genetic syndrome. During further follow-up of 5.04 (1.6-10.2) years no further additional procedures were needed. Conclusions: Pulmonary BVP of native pulmonary valve stenosis leads to excellent medium-term results, even in 3 of 4 infants with associated supravalvular obstruction sufficient pressure relief can be obtained.
\end{abstract}

\section{KEYWORDS}

Pediatric; catheter; congenital; pulmonary balloon valvuloplasty

\section{Introduction}

Pulmonary valve stenosis (PS) is a congenital heart defect (CHD) causing an obstruction of the right ventricular outflow tract at the valvular level. PS is common with an incidence of 0.7 per 1000 live births [1]. It may occur isolated or in combination with other types of CHD such as Tetralogy of Fallot with subvalvular or supravalvular pulmonary stenosis [2]. Depending on the hemodynamic severity, right ventricular pressure load leads to right ventricular myocardial hypertrophy. Clinical signs of PS include failure to thrive, exercise-induced dyspnea, arrhythmia or fatigue, but mostly young patients are

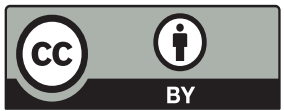

This work is licensed under a Creative Commons Attribution 4.0 International License, which permits unrestricted use, distribution, and reproduction in any medium, provided the original work is properly cited. 
oligosymptomatic [3]. In contrast, in newborns with critical PS with duct dependent pulmonary blood flow central cyanosis may be the leading symptom due to interatrial right-left-shunt [4]. Beside these clinical findings, the diagnosis of PS is made by transthoracic echocardiography and interventional and surgical treatment options are available for PS [3]. The catheter interventional technique of balloon valvuloplasty (BVP) has been introduced in 1982 by Kan et al. [5]. Nowadays, BVP of PS is the standard treatment for native PS in children [6-8] with acceptable results $[9,10]$.

Nevertheless, the occurrence of a residual PS after primary BVP during follow-up has been noticed $[8,11,12]$. One of the risk factors for residual PS includes the combination of valvular PS with an associated supravalvular obstruction [13]. Furthermore, the combination of valvular and supravalvular PS may also be associated with general hypoplasia of central and peripheral pulmonary arteries, often found in patients with genetic syndromes such as Allagile syndrome and Williams syndrome [14,15]. Controversy exists in literature about the preferred treatment for supravalvular PS, which can be either treated by surgery [16-18], or by balloon angioplasty of the main pulmonary artery [19-21]. Numbers of pediatric studies on treatment of combined valvular and supravalvular PS are limited.

Therefore, the aim of this study was to determine the medium-term follow-up of BVP of native PS in children and to identify possible factors for treatment failure leading to residual PS and need for secondary treatment.

Our hypothesis was that an additional supravalvular pulmonary stenosis, especially in children with genetic syndrome, may limit procedural success.

\section{Materials and Methods}

This study is a single centre, clinical observational study of children undergoing BVP for native PS. We reviewed the efficacy of BVP of native PS at our institution over the last 17 years for medium-term outcome with a focus on possible factors influencing outcome such as age at intervention, anatomic factors such as additional supravalvular stenosis, technical aspects of BVP, and genetic comorbidity. All consecutive patients with PS treated by BVP were identified within our digital hospital data base. The search was conducted with the keywords "pulmonary valve stenosis" and "balloon valvuloplasty" for the period from February 2002 to March 2019. Inclusion criteria were (1) age at intervention <19 years, (2) diagnosis of a native PS; including critical pulmonary stenosis, and (3) associated simple type of CHD, such as patent foramen ovale (PFO), atrial septal defect (ASD), small muscular ventricular septal defect (VSD) or patent arterial duct (PDA), all without hemodynamic relevance at the time of BVP. We further included neonates, who already were treated before birth by a fetal BVP of fetal PS. Critical PS was defined by duct-dependent pulmonary circulation and need of prostaglandin E2 infusion with or without severe cyanosis after birth.

Patients were excluded, if they had another preceding cardiac surgical procedure, or an associated complex type of CHD, such as Tetralogy of Fallot, double outlet right ventricle of Fallot type, pulmonary atresia with intact ventricular septum, or other CHD associated with valvular PS.

We did not exclude patients with Noonan syndrome, Williams' syndrome or other genetic syndromes associated with valvular PS.

The medical records, echocardiograms, angiography and cardiac catheterization data were reviewed.

The indication for BVP was based on the Doppler pressure gradient across the pulmonary valve. The indication for a BVP of PS was a right ventricular pressure increase of more than $2 / 3$ of the systolic arterial pressure in asymptomatic or more than $1 / 2$ of the systolic arterial pressure in symptomatic patients or duct-dependent pulmonary perfusion (critical PS).

\subsection{Catheter Procedure}

Transcatheter procedures were performed under general anaesthesia and endotracheal intubation. Standard technical approach was performed as previously described [22]. For BVP, the adequate balloon 
size was determined starting with a balloon diameter of at least $120 \%$ of the measured PV annulus in both projections. Balloon dilatation was performed with balloon inflation until the PV narrowing (balloon waist) disappeared, up to the recommended rated burst pressure. Post procedure, the peak-to-peak pressure gradient was re-measured across the pulmonary valve by a pressure curve pull back, and angiography of the PA and the RV were repeated. If a significant pressure gradient remained, secondary balloon valvuloplasty was performed with a larger balloon diameter (stepwise increase of balloon diameter by 1 to $2 \mathrm{~mm}$ ) and higher inflation pressures. The procedure was defined as successful, if a significant pressure gradient reduction defined as a residual peak-to-peak pressure gradient of less than $20 \mathrm{mmHg}$ or reduced systolic RV pressure less than half of the systemic arterial pressure and an improved opening of the PV with broader contrast jet on angiography was achieved. After cardiac catheterization, low molecular weight heparin was used twice daily for the following $24 \mathrm{~h}$ to prevent thromboembolic complications [23,24]. Patients stayed in hospital overnight for clinical observation and were discharged after clinical evaluation, electrocardiogram and echocardiogram one or two days after the procedure.

From the cardiac catheterization procedure, type of balloons, maximal balloon diameters and maximal balloon inflation pressures were noted. The maximal balloon diameter-to-PV annulus ratio, and the ratio between systolic RV pressure and systolic left ventricular pressure before and after the intervention were calculated. The SA pressure was evaluated before BVP. If the pressure of the LV was not noted in the protocol, systolic arterial blood pressure data was used. The diagnosis of supravalvular stenosis was made from angiography. Any complication during the procedure was noted and its severity was grouped according to Bergersen et al. [25].

\subsection{Doppler Echocardiography}

Two-dimensional transthoracic Doppler echocardiography, pulsatile and continuous wave Doppler and colour flow mapping were used to study cardiac anatomy, dimension, function and flow pattern. Three echocardiographic examinations were reviewed for each child at (1) the day before BVP, (2) the day after BVP and (3) at the time of last follow-up. For patients undergoing an additional procedure, the last follow-up is defined as the last echocardiography performed before reintervention. From these echocardiograms, the diameter of the PV annulus, the maximum peak gradient across the PV and the presence of pulmonary valvular regurgitation (PR) were noted. PR was assessed by colour-flow mapping and graded as none, mild, moderate and severe according to the ratio of diastolic colour Doppler jet width to outflow diameter of the RV. A ratio of jet width-to-RV outflow diameter gradient of less than $1 / 3$ was considered as mild, between $1 / 3$ to $2 / 3$ as moderate and of more than $2 / 3$ as severe PR [26].

\subsection{Statistics}

Continuous data were presented median with interquartile ranges (IQR). Categorial variables were expressed as numbers with percentages. For statistical evaluation of continuous variables, we used the paired $t$-test or Mann-Whitney U-Test depending on the type of distribution of assessed data. Categorial parameters were compared using the Pearson chi-square test and Fisher exact test. Kaplan-Meier analysis were used displaying freedom of additional procedures. A $p$-value $<0.05$ was determined as significant. All statistical analyses were performed using SPSS Statistics Version 25.0.

\subsection{Ethics}

The study was approved by the Cantonal Ethical Committee (BASEC Nr. 2019-01130).

\section{Results}

\subsection{Patients}

Pulmonary balloon valvuloplasty was performed in 218 patients between February 2002 and March 2019. We excluded patients with a former cardiac procedure other than BVP of PS $(n=40)$, associated 
complex CHD ( $\mathrm{n}=11)$, pulmonary atresia with intact ventricular septum who required catheter radiofrequency perforation of atretic PV before BVP $(\mathrm{n}=10)$ or missing informed study consent $(\mathrm{n}=14)$.

Therefore, we report on 143 patients fulfilling the inclusion criteria with native PS treated with BVP. 112 patients had an isolated valvular PS without additional supravavlular obstruction, 31 (21.7\%) patients had a combined valvular and supravalvular PS. Critical PS was found in 25 (17.5\%) neonates. Three patients were treated for PS before birth by a fetal BVP and needed further BVP for residual PS after birth.

Overall, the median (IQR) age at intervention was 2.6 (0.26-9.24) months, body weight 5.0 (3.4-8.0) kg, and body length $58(50-73) \mathrm{cm}$. Forty-seven of 143 patients (33\%) were neonates, 66 of $143(46 \%)$ were infants and 30 of $143(21 \%)$ were older than 1 year of age at BVP. Associated genetic syndromes were found in $10(7 \%)$ patients including Noonan syndrome $(\mathrm{n}=6)$, Williams' syndrome $(\mathrm{n}=2)$, Wolf-Hirschhorn syndrome $(\mathrm{n}=1)$, and Smith-Magenis syndrome $(\mathrm{n}=1)$. A total of $107(75 \%)$ infants had one or more additional minor CHD, including PFO $(\mathrm{n}=65$, among them $\mathrm{n}=29$ below 1 month of age), PDA $(\mathrm{n}=37$, among them $\mathrm{n}=25$ treated with prostaglandin E2 due to critical PS), ASD ( $n=42$ ASD II, treated during follow-up $n=4 ; n=1$ ASD I), small muscular VSD ( $\mathrm{n}=9$, treated during follow-up $\mathrm{n}=0$ ), and partial anomalous pulmonary venous drainage (PAPVD) $(\mathrm{n}=2$, treated during follow-up $\mathrm{n}=0)$. Combined procedures during initial BVP of PS were interventional closure of ASD II $(n=4)$, PFO $(n=1)$ and PDA $(n=1)$.

The immediate and medium-term results of BVP are shown in Table 1. Angiography revealed a median pulmonary annulus diameter comparable with the median diameter of the pulmonary valve annulus measured by echocardiography the day before $(8.1(7-10.4) \mathrm{mm}$ at cath $v s .8 .3(7-10) \mathrm{mm}$ on echo; $p=0.748)$. The used balloon diameter for BVP was $10.0(9-14) \mathrm{mm}$ resulting in balloon diameterto-PV annulus ratio of $1.28(1.2-1.4)$ with a maximal balloon pressure of 4 (3.5-10.0) atm. Further procedural details of BVP are given in Table 2.

Table 1: Baseline characteristics and medium-term results of balloon valvuloplasty of native PS in pediatric patients comparing valvular and valvular + supravalvular PS

\begin{tabular}{|c|c|c|c|c|}
\hline & \multirow[b]{2}{*}{$\begin{array}{l}\text { Total patients } \\
(\mathrm{n}=143)\end{array}$} & \multicolumn{2}{|c|}{ Pulmonary valve stenosis } & \multirow[b]{2}{*}{$p$ value } \\
\hline & & $\begin{array}{l}\text { Valvular } \\
(\mathrm{n}=112)\end{array}$ & $\begin{array}{l}\text { Valvular + supravalvular }(\mathrm{n} \\
=31)\end{array}$ & \\
\hline Age [years] & $0.22(0.02-0.8)$ & $0.14(0.01-0.7)$ & $0.47(0.2-1.2)$ & 0.002 \\
\hline Sex, female $[\mathrm{n}=, \%]$ & $83.0(58 \%)$ & $68.0(60.7 \%)$ & $15.0(48.4 \%)$ & 0.218 \\
\hline Body weight $[\mathrm{kg}]$ & $5.0(3.4-8.0)$ & $4.6(3.2-7.7)$ & $6.3(4.7-9.2)$ & 0.033 \\
\hline Body length $[\mathrm{cm}]$ & $58.0(50.0-73.0)$ & $55.5(50.0-70.0)$ & $61.0(56.0-75.0)$ & 0.580 \\
\hline \multicolumn{5}{|l|}{ Catheterization } \\
\hline Balloon-to-PV annulus ratio & $1.28(1.2-1.4)$ & $1.25(1.2-1.3)$ & $1.4(1.3-1.5)$ & $<0.001$ \\
\hline Max balloon pressure $[\mathrm{atm}]$ & $4.0(3.5-10.0)$ & $4.0(3.5-6)$ & $10.0(7.0-14.0)$ & $<0.001$ \\
\hline Max balloon diameter $[\mathrm{mm}]$ & $10.0(9.0-14.0)$ & $10.0(8.0-12.0)$ & $12.0(10.0-14.0)$ & 0.005 \\
\hline Used balloons (per patient) $[\mathrm{n}=]$ & 209 (= 1.5/pat.) & 147 (= 1.3/pat.) & 62 (= 2/pat.) & $<0.001$ \\
\hline Low pressure balloon $(\leq 4 \mathrm{~atm})[\mathrm{n}=]$ & $95.0(66.4 \%)$ & $84.0(75.0 \%)$ & $11.0(35.5 \%)$ & $<0.001$ \\
\hline High pressure balloon $(>4 \mathrm{~atm})[\mathrm{n}=]$ & $48.0(33.6 \%)$ & $28.0(25.0 \%)$ & $20.0(64.5 \%)$ & $<0.001$ \\
\hline Procedure time [min] & $50.0(39.3-63.8)$ & $46.5(37.0-58.0)$ & $79.5(56.0-94.8)$ & $<0.001$ \\
\hline X-ray time $[\mathrm{min}]$ & $11.3(7.0-15.2)$ & $10.0(6.6-14.0)$ & $15.15(11.1-24.3)$ & 0.001 \\
\hline X-ray dose $[\mathrm{cGy} / \mathrm{m} 2]$ & $0.9(0.44-2.3)$ & $0.8(0.3-1.95)$ & $1.1(0.6-4.5)$ & 0.107 \\
\hline Contrast medium [ml] & $18.0(12-30)$ & $16.0(12-24)$ & $30.0(18.0-42.0)$ & $<0.001$ \\
\hline Systolic dp RV-PA, before [mmHg] & $43.5(31.3-59.0)$ & $42.0(30.3-59.0)$ & $45.0(32.0-58.3)$ & 0.756 \\
\hline
\end{tabular}


Table 1 (continued)

\begin{tabular}{|c|c|c|c|c|}
\hline & \multirow[b]{2}{*}{$\begin{array}{l}\text { Total patients } \\
(\mathrm{n}=143)\end{array}$} & \multicolumn{2}{|c|}{ Pulmonary valve stenosis } & \multirow[b]{2}{*}{$p$ value } \\
\hline & & $\begin{array}{l}\text { Valvular } \\
(\mathrm{n}=112)\end{array}$ & $\begin{array}{l}\text { Valvular + supravalvular }(\mathrm{n} \\
=31)\end{array}$ & \\
\hline Systolic dp RV-PA, after [mmHg] & $14.0(9.0-20.0)$ & $12.0(8.0-17.0)$ & $28.5(15.8-40)$ & $<0.001$ \\
\hline Systolic RV pressure, before $[\mathrm{mmHg}]$ & $64.0(52.0-84.0)$ & $66.0(52.0-86.0)$ & $63.0(52.0-78.0)$ & 0.357 \\
\hline Systolic PA pressure, before $[\mathrm{mmHg}]$ & $20.0(17.0-27.5)$ & $20.5(17.0-28.0)$ & $20.0(16.0-25.0)$ & 0.420 \\
\hline Systolic RV pressure, after [mmHg] & $41.0(35.0-51.0)$ & $39.0(33.0-47.0)$ & $52.5(42.5-60.8)$ & $<0.001$ \\
\hline Systolic PA pressure, after $[\mathrm{mmHg}]$ & $25.0(21.0-32.0)$ & $26.0(21.0-33.0)$ & $23.0(18.5-29.0)$ & 0.094 \\
\hline Systolic SA pressure, before $[\mathrm{mmHg}]$ & $74.0(62.0-85.0)$ & $73.0(60.0-85.0)$ & $78.5(66.3-87.5)$ & 0.178 \\
\hline RV-to-SA pressure, before & $0.9(0.7-1.2)$ & $0.95(0.7-1.4)$ & $0.86(0.6-1.04)$ & 0.179 \\
\hline \multicolumn{5}{|l|}{ Echocardiography } \\
\hline $\begin{array}{l}\text { Peak Doppler dP RV-PA, before } \\
{[\mathrm{mmHg}]}\end{array}$ & $68.0(57.0-81.0)$ & $68.5(57.3-81.8)$ & $65.0(53.0-80.0)$ & 0.559 \\
\hline $\begin{array}{l}\text { Peak Doppler dP RV-PA, } 1 \text { day after } \\
{[\mathrm{mmHg}]}\end{array}$ & $25.0(19.0-45.0)$ & $23.0(18.0-29.0)$ & $54.0(45.0-62.0)$ & $<0.001$ \\
\hline $\begin{array}{l}\text { Peak Doppler dP RV-PA, last FU } \\
{[\mathrm{mmHg}]}\end{array}$ & $14.0(5.8-22.0)$ & $13.0(5.0-21.0)$ & $25.0(10.5-65.5)$ & 0.007 \\
\hline PV annulus $[\mathrm{mm}]$ & $8.3(7.0-10.0)$ & $8.0(7.0-10.1)$ & $9.0(8.0-10.3)$ & 0.046 \\
\hline $\mathrm{PV}$ regurgitation, $1 \mathrm{~d}$ after $[\mathrm{n}=, \%]$ & $115(80.4 \%)$ & $100(89.3 \%)$ & $15(48.4 \%)$ & $<0.001$ \\
\hline No $[\mathrm{n}=, \%]$ & $28(19.6 \%)$ & $12(10.7 \%)$ & $16(51.6 \%)$ & $<0.001$ \\
\hline Mild $[\mathrm{n}=, \%])$ & $98(68.5 \%)$ & $86(76.9 \%)$ & $12(38.7 \%)$ & $<0.001$ \\
\hline Moderate $[\mathrm{n}=, \%]$ & $16(11.2 \%)$ & $13(11.6 \%)$ & $3(9.7 \%)$ & 1.000 \\
\hline Severe $[\mathrm{n}=, \%]$ & $1(0.7 \%)$ & $1(0.9 \%)$ & $0(0 \%)$ & 1.000 \\
\hline $\mathrm{PV}$ regurgitation, last $\mathrm{FU}[\mathrm{n}=, \%]$ & $111(90.9 \%)$ & $94(95.9 \%)$ & $17(70.8 \%)$ & 0.001 \\
\hline No $[\mathrm{n}=, \%]$ & $11(9.0 \%)$ & $4(4.1 \%)$ & $7(29.2 \%)$ & 0.001 \\
\hline Mild PR $[\mathrm{n}=, \%]$ & $78(63.9 \%)$ & $62(63.3 \%)$ & $16(66.7 \%)$ & 0.941 \\
\hline Moderate PR $[\mathrm{n}=, \%]$ & $27(22.1 \%)$ & $26(26.5 \%)$ & $1(4.2 \%)$ & 0.037 \\
\hline Severe PR $[\mathrm{n}=, \%]$ & $6(4.9 \%)$ & $6(6.1 \%)$ & $0(0.0 \%)$ & 0.597 \\
\hline \multicolumn{5}{|l|}{ Follow-up } \\
\hline Reintervention $[\mathrm{n}=, \%]$ & $14(9.8 \%)$ & $6(5.4 \%)$ & $8(25.8 \%)$ & 0.002 \\
\hline Complications $[\mathrm{n}=, \%]$ & $8(5.6 \%)$ & $7(6.3 \%)$ & $1(3.2 \%)$ & 1.000 \\
\hline
\end{tabular}

Note: Data are given as numbers (\%), and median (IQR). dP, systolic pressure gradient; PS, pulmonary valve stenosis; PV, pulmonary valve; RV, right ventricle; SA, systemic artery (Aorta).

At catheterization, we found a significant reduction of invasive measured systolic RV pressure (before BVP 64.0 (52.0-84.0) $\mathrm{mmHg} v$ s. after BVP $41.0(35.0-51.0) \mathrm{mmHg} ; p<0.001)$, and systolic RV-PA pressure gradient (before 43.5 (31.3-59.0) $\mathrm{mmHg} v s$. after $14.0(9.0-20.0) \mathrm{mmHg} ; p<0.001)$ and increase of systolic PA pressure (before BVP 20.0 (17.0-27.5) mmHg vs. after BVP 25.0 (21.0-32.0) mmHg; $p<0.001$ ). Before BVP, the ratio systolic RV pressure-to-systolic arterial pressure was 0.9 (0.7-1.2) (Table 1).

Immediate and medium-term results of BVP of PS determined by echocardiography are shown in Fig. 1. The peak Doppler pressure gradient was reduced from 68.0 (57.0-81.0) $\mathrm{mmHg}$ before BVP to 25.0 (19.045.0) $\mathrm{mmHg}$ on the day after BVP and $14.0(5.8-22.0) \mathrm{mmHg}$ at last follow-up (all $p<0.001$ ). 
Table 2: Procedural details of BVP of native PS in pediatric patients

\begin{tabular}{ll}
\hline Sheath size, maximal [French] & $5(4-14)$ \\
\hline Number of needed balloons $[\mathrm{n}=, \%]$ & \\
1 & $89(62.2 \%)$ \\
2 & $45(31.5 \%)$ \\
3 or more & $9(6.3 \%)$ \\
Used balloons & \\
Tyshak II® $[\mathrm{n}=]$ & 84 \\
Tyshak mini ${ }^{\circledR}[\mathrm{n}=]$ & 19 \\
Atlas ${ }^{\circledR}[\mathrm{n}=]$ & 15 \\
Advance ${ }^{\circledR} 18$ LP $[\mathrm{n}=]$ & 16 \\
Other* $[\mathrm{n}=]$ & 75 \\
\hline
\end{tabular}

Note: Data are given as numbers (\%), and median (range) [IQR], as appropriate. BVP, balloon valvuloplasty; PS, pulmonary valve stenosis. *Other included Coefficient ${ }^{\mathrm{TM}}(\mathrm{n}=15)$, VASC $($ II $(\mathrm{n}=7), Z$-med ${ }^{\mathrm{TM}} \mathrm{II}(\mathrm{n}=6)$, Maxi $\mathrm{LD}^{\mathrm{TM}}(\mathrm{n}=4)$, Sterling ${ }^{\mathrm{TM}}(\mathrm{n}=3)$, Conquest $\mathbb{R}(\mathrm{n}=1)$, Dorado ${ }^{\circledR}(n=1)$, Powerflex $(n=1)$, and unknown $(n=37)$.

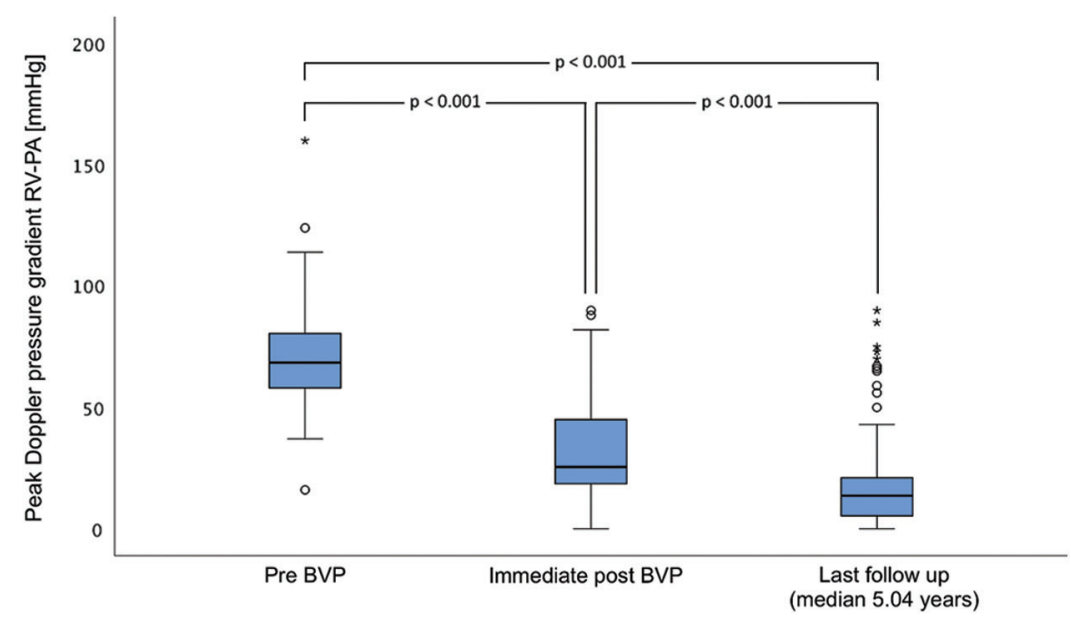

Figure 1: Medium-term results of echocardiographic peak Doppler pressure gradient of balloon valvuloplasty of native pulmonary valve stenosis in pediatric patients. BVP, balloon valvuloplasty; FU, follow-up; PA, pulmonary artery; RV, right ventricle. Of note, the outliers depicted as asterisks and dots according to supravalvular and valvular pulmonary valve stenosis at the last follow-up needed reintervention

The follow-up was 5.04 (1.6-0.2) years. Twenty-one patients were lost of follow-up and were excluded for medium-term analysis (Fig. 1). Follow up rate was $85.4 \%$.

The distribution of the degree of PR determined on echo at the day after pulmonary BVP were in more than two thirds of the patients of mild degree $(n=98,69 \%)$ and remained stable during medium-term followup $(\mathrm{n}=78,64 \%)$ (Fig. 2). PR after pulmonary BVP of PS was not associated with oversizing of balloons used during BVP procedures. In 26 of $143(18.2 \%)$ patients the balloon diameter-to-PV annulus ratio exceeded more than 1.4 but did not result in a higher degree of PR on the day after BVP or at last follow-up. PV replacement for PR was not necessary during medium-term follow-up. 


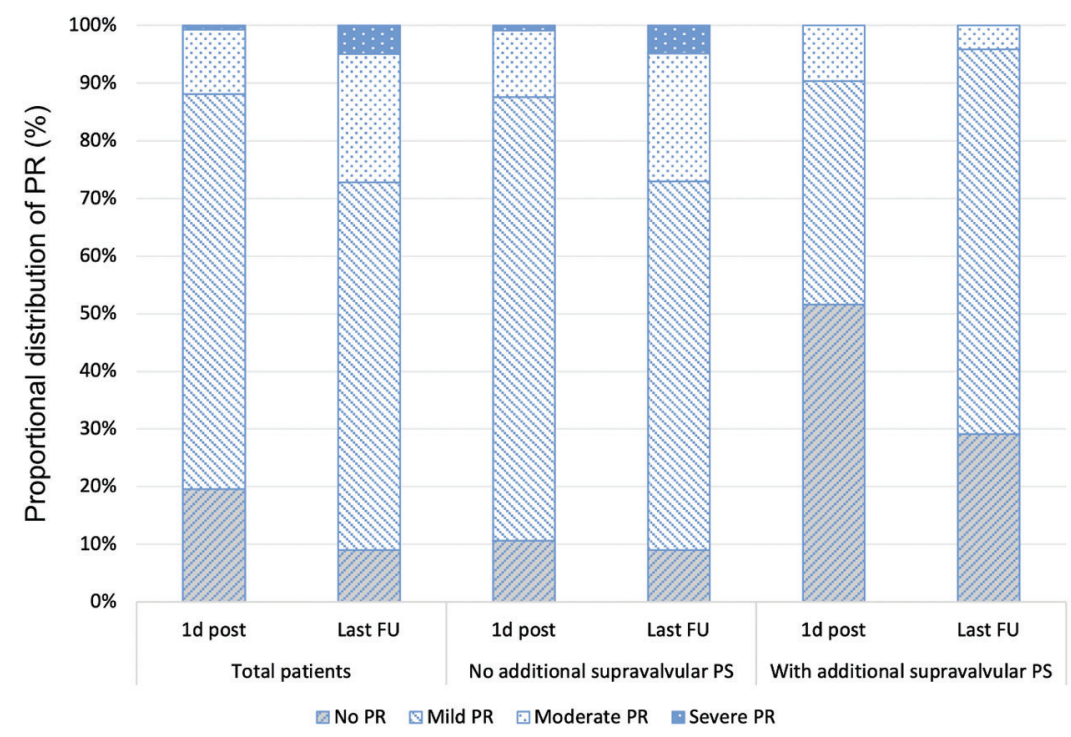

Figure 2: Medium-term results of pulmonary valve regurgitation after balloon valvuloplasty of native pulmonary valve stenosis with and without additional supravalvular pulmonary stenosis in pediatric patients. $1 \mathrm{~d}$ post, day after; last FU, last follow-up; PR, pulmonary valve regurgitation; PS, pulmonary valve stenosis

\subsection{Combined Valvular and Supravalvular Pulmonary Stenosis}

Patients with a combined valvular and supravalvular PS $(n=31,21.7 \%)$, were older age at BVP ( 0.47 years $v s .0 .14$ years; $p=0.002)$, had more complex procedures with longer median procedure times (79.5 min vs. $46.5 \mathrm{~min} ; p<0.001$ ), larger balloon-to-PV annulus ratios $(1.4 v s .1 .25 ; p<0.001)$, more frequent use of high-pressure balloons $(64.5 \%$ vs. $25 \% ; p<0.001)$ and the resulting residual pressure gradients at cath $(28.5(15.8-40) \mathrm{mmHg} v s .12 .0(8-17) \mathrm{mmHg} ; p<0.001)$ and on echo the day after the procedure (54.0 (45-62) mmHg vs. $23.0(18-19) \mathrm{mmHg} ; p<0.001)$ were higher (Table 1). Furthermore, patients with an additional supravalvular PS had more often associated genetic syndromes $(23 \% v s .3 \%$; $p<0.001)$. Of note, PR was less frequently found in patients with additional supravalvular PS immediately after BVP and at last follow-up (48\% vs. 89\% and 71\% vs. 96\%; both $p<0.001)$ (Fig. 2). Eight of the 31 patients (25.8\%) with additional supravalvular PS needed additional procedures (Table 1 ).

\subsection{Complications of $\mathrm{BVP}$}

In eight patients $(5.6 \%)$ complications during or after BVP occurred, determined as minor $(\mathrm{n}=4)$, moderate $(\mathrm{n}=3)$, and severe $(\mathrm{n}=1)$. Minor complications included transient arrhythmias during BVP $(\mathrm{n}=3)$, and intraprocedural small local femoral vein bleeding complication during sheath exchange $(\mathrm{n}=$ 1), both complications were self-limiting and needed no further treatment. Moderate complications were supraventricular tachycardia treated by adenosine application $(n=1)$, infundibular RV muscle spasm treated by beta-blocking agent during BVP $(n=1)$ and a vessel injury of the right femoral vein, which was closed by detachable coils $(\mathrm{n}=1)$. Major complication occurred in a neonate 30 days after BVP with infective endocarditis of the PV due to Staphylococcus aureus $(\mathrm{n}=1)$. The PV had to be replaced by surgery after six weeks due to a paravalvular abscess formation. Three children died during further follow-up-none of these deaths were procedure-related. The reasons for death were necrotizing enterocolitis (5 weeks after BVP due to critical PS), ponsglioma (6 years after BVP), and postoperative sudden cardiac death (6 weeks after secondary surgery for supravalvular PS). 


\subsection{Additional Procedures-Interventional or Surgical}

Freedom of additional procedures was $90.2 \%$ after 1.4 years for the total patient group, $74.2 \%$ for patients with and $94.6 \%$ for patients without additional supravalvular PS and did not change during further medium-term follow-up (Fig. 3). Additional procedures became necessary in 14 patients (9.8\%) after $0.2(0.1-0.7)$ years (Table 1$)$. Reasons for additional procedures were residual PS $(\mathrm{n}=13)$ and infective endocarditis of PV $(n=1)$. Residual PS was localised at valvular $(n=3)$, subvalvular $(n=2)$, or supravalvular $(\mathrm{n}=8)$ level. Additional procedures for PV restenosis were performed by cardiac surgery ( $n=7$, among them $n=6$ due to supravalvular PS) or by a second $(n=5)$ or a third $(n=1)$ BVP procedure. One patient was operated for surgical ASD closure, but also received an additional pulmonary valvotomy, which by itself would not have been indicated due to low residual RV-to-PA pressure gradient (24 mmHg).

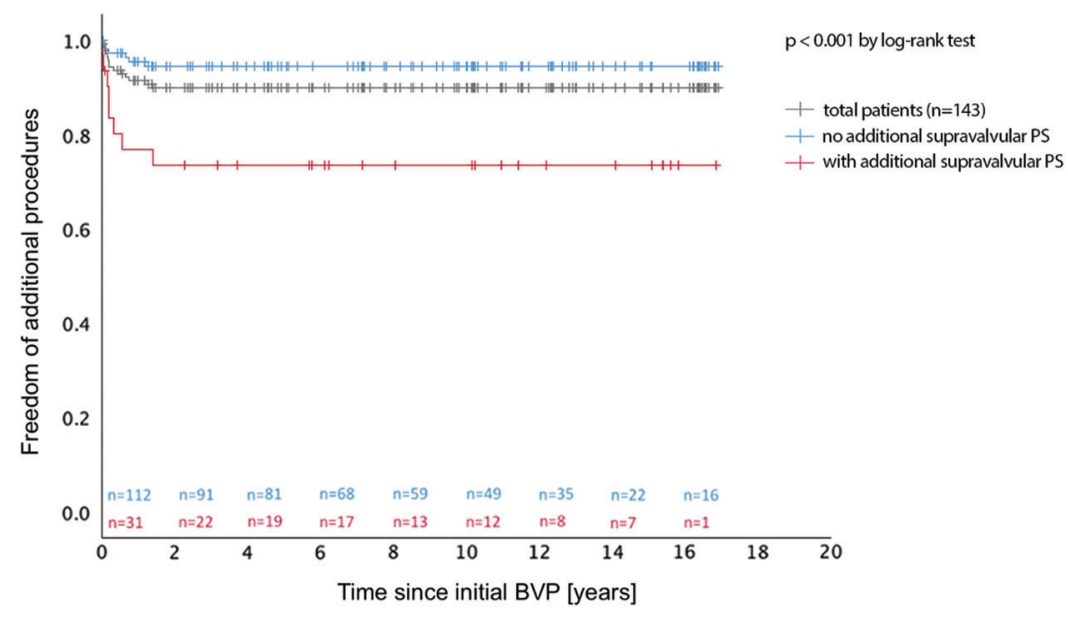

Figure 3: Freedom of additional procedure after pulmonary balloon valvuloplasty with and without additional supravalvular pulmonary stenosis in children. BVP, balloon valvuloplasty; PS, pulmonary valve stenosis

Factors determining an additional procedure were an associated supravalvular PS $(p=0.002)$ resulting in a higher immediate residual pressure gradient at cath $(p<0.001)$. These findings were also associated with a prolonged procedure time of cardiac catheterization $(p=0.006)$, a higher number of balloons used $(p<$ $0.001)$ and a higher peak Doppler pressure gradient on echocardiography $(p<0.001)$ on the day after procedure. We did not find any influence of associated genetic syndromes for additional procedures after BVP.

\section{Discussion}

This data analysis of the medium-term results of BVP in native PS revealed excellent technical efficacy, high procedural safety, and low complication rate as well as a long-lasting efficacy during follow-up (Fig. 1). These findings are according to the literature [9-11,27].

Despite an overall freedom of additional procedures of more than $90 \%$ over a follow-up of more than five years, patients needed reintervention early during follow-up until 1.4 years after initial BVP (Fig. 3). Karagoz et al. reported procedural success as time of freedom from reintervention for one year after BVP [27]. Our rate of additional procedures is comparable with other groups [8,11,12]. Residual PS was the most frequent reason for additional procedures, while PR did not lead to reintervention and may only mildly develop during followup (Fig. 2). In patients with residual PS, secondary BVP was the treatment of choice for valvular PS and was successful, but in patients with combined valvular and supravalvular restenosis, surgery was performed, as recommended by the AHA [16,22]. While the efficacy of BVP in children with isolated valvular PS is 
excellent, pulmonary BVP has limitations in children with a combined valvular and supravalvular PS [21], attributing to the high number of additional procedures needed in our cohort (Table 1). The number of additional supravalvular PS was high (21.7\%), requiring more aggressive procedural BVP, but with a comparable low number of complications and good success rate of BVP (23 of 31 patients, 74\%). Nevertheless, supravalvular PS has been described as risk factor for procedural failure [13,21] and its absence as a significant predictor of success [28]. Surgery is mentioned as the recommended first line therapy for supravalvular PS [16,22], because only few studies report successful BVP in these patients [1921]. The elastic recoil properties of the supravalvular ridge were discussed as one possible contributing factor to the failure of BVP in patients with supravalvular PS [18].

From our perspective, supravalvular PS can be treated by pulmonary balloon angioplasty with a procedural success rate of almost $75 \%$, which is in comparison to neonatal cardiac surgery a less invasive procedure in small children [8]. Our findings are comparable to a recently published trial where the reintervention rate was $32 \%$ in patients with supravalvular PS. Poupart et al. [21] A higher balloon-to-PV annulus up to 1.4 can be used without a higher rate of complications (Table 1). Of note, the number of PR is lower in patients with combined valvular and supravalvular PS, which underlines the more relevant aspect of the supravalvular PS (Table 1). Further studies need to investigate which patients with supravalvular PS might be successfully treated by BVP and which might not [21].

An immediate residual systolic RV-to-PA gradient after BVP of valvular PS of $<20 \mathrm{mmHg}$ may be determined as procedural success [16], while other studies [12,13,22,29] determined higher cut-off values (25 to $36 \mathrm{mmHg}$ ) with no need for reintervention during mid-term follow-up [22]. Nevertheless, more aggressive BVP may lead to higher complication rates including PR [8,30], which was not found in our cohort, even in those children with higher balloon-to-PV annulus ratio $>1.4$ (Table 1).

PR after BVP may develop and progress during medium-term follow-up, but the hemodynamic impact of PR remains rather low [9] due to the mild-to-moderate degree of PR in most cases during long-term follow-up $[9,10,12]$. The reasons for developing severe PR after BVP and during long-term follow-up are unclear, a higher balloon diameter-to-PV annulus ( $>1.4)$ has been discussed as a risk factor [12,30-32]. Nevertheless, PR rarely leads to surgical valve replacement [11,30,32,33]. We recommend critical evaluation of the RV function and RV volume during follow-up including multi-modal imaging [33].

The very low number of severe complications $(<1 \%)$ in our cohort underlines the technical safety of the procedure $[10,13,22]$. In our series, PV infective endocarditis after BVP of PS was attributed to central venous line catheter sepsis with Staphylococcus aureus in an otherwise already critical ill neonate, but remains a rather anecdotal complication [34,35], where peri-procedural antibiotic endocarditis prophylaxis is no more recommended in patients undergoing BVP of PS [3].

Suboptimal outcome of BVP of PS in patients with Noonan syndrome has been reported [12]. In our cohort, only $1 / 10$ of the patients with a genetic syndrome needed additional procedures, namely one with Noonan syndrome, while the need of reintervention in patients with Noonan syndrome after pulmonary BVP differs to more frequent findings of other groups [36].

Former fetal BVP of PS may lead to the necessity of further BVP of PS after birth, as shown in our cohort, performed with a high rate of success, as described by Tulzer et al. [37].

\section{Limitations}

The main limitation of this data collection and analysis was the retrospective study design, which made standardized follow-up difficult. Furthermore, follow-up of echocardiographic results is dependent on subjective judgment of the examiner and is therefore difficult to standardise. Nowadays, as an alternative for using echocardiography within our retrospective analysis, cardiac MRI as part of multimodality imaging techniques has become the diagnostic standard for evaluating pulmonary regurgitation and right 
ventricular volume load, which should be considered for future studies [38]. Nevertheless, recent studies have shown that echocardiography is still an appropriate technique for the assessment of PR [39].

\section{Conclusion}

BVP of isolated native PS is safely performed with an excellent medium-term success and a high rate of freedom from additional procedures. The main factor for additional procedure was an associated supravalvular PS resulting in a higher residual pressure gradient. Patients with additional supravalvular PS can be treated with BVP in a first attempt, since BVP was successful in almost 3 out of 4 of these patients $(74.2 \%)$ and BVP is less invasive than surgery. Further studies, preferably prospective, are needed to better understand the circumstances for success of BVP in patients with supravalvular PS.

Acknowledgement: The authors thank the entire catheter laboratory team and colleagues involved in the follow-up, and Ms. Silvia Hilfiker for her assistance in study coordination, Ms. Sandra Siegfried for statistical supervision and the referring cardiologists for abandon the follow-up findings.

Funding Statement: The authors received no specific funding for this study.

Conflicts of Interest: The authors declare that they have no conflicts of interest to report regarding the present study.

\section{References}

1. Hoffman, J. I., Kaplan, S. (2002). The incidence of congenital heart disease. Journal of the American College of Cardiology, 39(12), 1890-1900.

2. Baumgartner, H., Hung, J., Bermejo, J., Chambers, J. B., Evangelista, A. et al. (2009). Echocardiographic assessment of valve stenosis: EAE/ASE recommendations for clinical practice. Journal of the American Society of Echocardiography, 22(1), 1-102.

3. Cuypers, J. A., Witsenburg, M., van der Linde, D., Roos-Hesselink, J. W. (2013). Pulmonary stenosis: Update on diagnosis and therapeutic options. Heart, 99(5), 339-347.

4. Loureiro, P., Cardoso, B., Gomes, I. B., Martins, J. F., Pinto, F. F. (2017). Long-term results of percutaneous balloon valvuloplasty in neonatal critical pulmonary valve stenosis: A 20-year, single-centre experience. Cardiology in the Young, 27(7), 1314-1322.

5. Kan, J. S., White, R. I., Mitchell, S. E., Gardner, T. J. (1982). Percutaneous balloon valvuloplasty: A new method for treating congenital pulmonary-valve stenosis. New England Journal of Medicine, 307(9), 540-542.

6. Schmaltz, A. A., Bein, G., Grävinghoff, L., Hagel, K., Hentrich, F. et al. (1989). Balloon valvuloplasty of pulmonary stenosis in infants and children-co-operative study of the German Society of Pediatric Cardiology. European Heart Journal, 10(11), 967-971.

7. O’Connor, B. K., Beekman, R. H., Lindauer, A., Rocchini, A. (1992). Intermediate-term outcome after pulmonary balloon valvuloplasty: Comparison with a matched surgical control group. Journal of the American College of Cardiology, 20(1), 169-173.

8. Peterson, C., Schilthuis, J. J., Dodge-Khatami, A., Hitchcock, J. F., Meijboom, E. J. et al. (2003). Comparative long-term results of surgery versus balloon valvuloplasty for pulmonary valve stenosis in infants and children. Annals of Thoracic Surgery, 76(4), 1078-1083.

9. Voet, A., Rega, F., de Bruaene, A. V., Troost, E., Gewillig, M. et al. (2012). Long-term outcome after treatment of isolated pulmonary valve stenosis. International Journal of Cardiology, 156(1), 11-15.

10. Parent, J. J., Ross, M. M., Bendaly, E. A., Breinholt, J. P. (2017). Results of pulmonary balloon valvuloplasty persist and improve at late follow-up in isolated pulmonary valve stenosis. Cardiology in the Young, 27(8), 1566-1570.

11. Rao, P. S., Galal, O., Patnana, M., Buck, S. H., Wilson, A. D. (1998). Results of three to 10 year follow up of balloon dilatation of the pulmonary valve. Heart, 80(6), 591-595. 
12. McCrindle, B. W. (1994). Independent predictors of long-term results after balloon pulmonary valvuloplasty. Valvuloplasty and Angioplasty of Congenital Anomalies (VACA) Registry Investigators. Circulation, 89(4), 1751-1759.

13. Holzer, R. J., Gauvreau, K., Kreutzer, J., Trucco, S. M., Torres, A. et al. (2012). Safety and efficacy of balloon pulmonary valvuloplasty: A multicenter experience. Catheterization and Cardiovascular Interventions: Official Journal of the Society for Cardiac Angiography \& Interventions, 80(4), 663-672.

14. McElhinney, D. B., Krantz, I. D., Bason, L., Piccoli, D. A., Emerick, K. M. et al. (2002). Analysis of cardiovascular phenotype and genotype-phenotype correlation in individuals with a JAG1 mutation and/or Alagille syndrome. Circulation, 106(20), 2567-2574.

15. Scheiber, D., Fekete, G., Urban, Z., Tarjan, I., Balaton, G. et al. (2006). Echocardiographic findings in patients with Williams-Beuren syndrome. Wiener Klinische Wochenschrift, 118(17-18), 538-542.

16. Warnes, C. A., Williams, R. G., Bashore, T. M., Child, J. S., Connolly, H. M. et al. (2008). ACC/AHA, 2008 guidelines for the management of adults with congenital heart disease: Executive summary: A report of the American college of cardiology/American heart association task force on practice guidelines (writing committee to develop guidelines for the management of adults with congenital heart disease). Circulation, $118(23), 2395-2451$.

17. Hong, D., Qian, M. Y., Zhang, Z. W., Wang, S. S., Li, J. J. et al. (2017). Immediate therapeutic outcomes and medium-term follow-up of percutaneous balloon pulmonary valvuloplasty in infants with pulmonary valve stenosis: A single-center retrospective study. Chinese Medical Journal, 130(23), 2785-2792.

18. Bacha, E. A., Kalimi, R., Starr, J. P., Quinones, J., Koenig, P. (2004). Autologous repair of supravalvar pulmonic stenosis. Annals of Thoracic Surgery, 77(2), 734-736.

19. Kodama, K., Owa, M., Saito, M., Katsuki, T., Omura, N. et al. (1992). A case of adult pulmonary supravalvular membranous stenosis which was successfully treated by percutaneous transluminal balloon dilatation. Respiration \& Circulation, 40(10), 1025-1029.

20. Rao, P. S. (1992). Transcatheter treatment of pulmonary outflow tract obstruction: A review. Progress in Cardiovascular Diseases, 35(2), 119-158.

21. Poupart, S., Navarro-Castellanos, I., Raboisson, M. J., Lapierre, C., Dery, J. et al. (2021). Supravalvular and valvular pulmonary stenosis: Predictive features and responsiveness to percutaneous dilation. Pediatric Cardiology, 42(4), 814-820.

22. Rao, P. S. (2007). Percutaneous balloon pulmonary valvuloplasty: State of the art. Catheterization and Cardiovascular Interventions: Official Journal of the Society for Cardiac Angiography \& Interventions, 69(5), 747-763.

23. Cohen, C. T., Anderson, V., Desai, S. B., Arunachalam, A., Ahmed, M. et al. (2021). Patient characteristics and treatment outcomes of symptomatic catheter-related arterial thrombosis in infants: A retrospective cohort study. Journal of Pediatrics, 231, 215-222.

24. Pardun, N., Lemmer, J., Belker, K., Pringsheim, M., Ewert, P. et al. (2021). Low-molecular-weight heparin administered by subcutaneous catheter is a safe and effective anti-coagulation regimen in selected inpatient infants and children with complex congenital heart disease. Cardiology in the Young, 16, 1-6 (Epub ahead of print). DOI 10.1017/S1047951121000317.

25. Bergersen, L., Giroud, J. M., Jacobs, J. P., Franklin, R. C., Béland, M. J. et al. (2011). Report from the international society for nomenclature of paediatric and congenital heart disease: Cardiovascular catheterisation for congenital and paediatric cardiac disease (Part 2 - Nomenclature of complications associated with interventional cardiology). Cardiology in the Young, 21(3), 260-265.

26. Srivastava, S., Parness, I. A. (2009). Tetralogy of Fallot. Echocardiography in pediatric and congenital heart disease: From fetus to adult, pp. 362-384. Oxford: Wiley-Blackwell.

27. Karagoz, T., Asoh, K., Hickey, E., Chaturvedi, R., Lee, K. J. et al. (2009). Balloon dilation of pulmonary valve stenosis in infants less than $3 \mathrm{~kg}$ : A 20-year experience. Catheterization and Cardiovascular Interventions: Official Journal of the Society for Cardiac Angiography \& Interventions, 74(5), 753-761.

28. El-Saeidi, S. A., Hamza, H. S., Agha, H. M., Soliman, M. M., Attia, W. A. et al. (2020). Experience with balloon pulmonary valvuloplasty and predictors of outcome: A ten-year study. Cardiology in the Young, 30(4), 482-488 (Epub 2020 Feb 19). DOI 10.1017/S1047951120000360. 
29. Gudausky, T. M., Beekman, R. H. (2006). Current options, and long-term results for interventional treatment of pulmonary valvar stenosis. Cardiology in the Young, 16(5), 418-427.

30. Berman, W., Fripp, R. R., Raisher, B. D., Yabek, S. M. (1999). Significant pulmonary valve incompetence following oversize balloon pulmonary valveplasty in small infants: A long-term follow-up study. Catheterization and Cardiovascular Interventions: Official Journal of the Society for Cardiac Angiography \& Interventions, 48(1), 61-66.

31. Poon, L. K., Menahem, S. (2003). Pulmonary regurgitation after percutaneous balloon valvoplasty for isolated pulmonary valvar stenosis in childhood. Cardiology in the Young, 13(5), 444-450.

32. Garty, Y., Veldtman, G., Lee, K., Benson, L. (2005). Late outcomes after pulmonary valve balloon dilatation in neonates, infants and children. Journal of Invasive Cardiology, 17(6), 318-322.

33. Devanagondi, R., Peck, D., Sagi, J., Donohue, J., Yu, S. et al. (2017). Long-term outcomes of balloon valvuloplasty for isolated pulmonary valve stenosis. Pediatric Cardiology, 38(2), 247-254.

34. Kalra, G. S., Wander, G. S., Anand, I. S. (1990). Right sided endocarditis after balloon dilatation of the pulmonary valve. British Heart Journal, 63(6), 368-369.

35. Santoro, G., Formigari, R., Carlo, D. D., Pasquini, L., Ballerini, L. (1995). Midterm outcome after pulmonary balloon valvuloplasty in patients younger than one year of age. American Journal of Cardiology, 75(8), 637-639.

36. Prendiville, T. W., Gauvreau, K., Tworog-Dube, E., Patkin, L., Kucherlapati, R. S. et al. (2014). Cardiovascular disease in Noonan syndrome. Archives of Disease in Childhood, 99(7), 629-634.

37. Tulzer, A., Arzt, W., Gitter, R., Prandstetter, C., Grohmann, E. et al. (2018). Immediate effects and outcome of inutero pulmonary valvuloplasty in fetuses with pulmonary atresia with intact ventricular septum or critical pulmonary stenosis. Ultrasound in Obstetrics \& Gynecology: The Official Journal of the International Society of Ultrasound in Obstetrics and Gynecology, 52(2), 230-237.

38. Valente, A. M., Cook, S., Festa, P., Ko, H. H., Krishnamurthy, R. et al. (2014). Multimodality imaging guidelines for patients with repaired tetralogy of fallot: A report from the AmericanSsociety of Echocardiography: Developed in collaboration with the Society for Cardiovascular Magnetic Resonance and the Society for Pediatric Radiology. Journal of the American Society of Echocardiography: Official Publication of the American Society of Echocardiography, 27(2), 111-141.

39. Dellas, C., Kammerer, L., Gravenhorst, V., Lotz, J., Paul, T. et al. (2018). Quantification of pulmonary regurgitation and prediction of pulmonary valve replacement by echocardiography in patients with congenital heart defects in comparison to cardiac magnetic resonance imaging. International Journal of Cardiovascular Imaging, 34(4), 607-613. 